\title{
From protocol to product: ventral midbrain dopaminergic neuron differentiation for the treatment of Parkinson's disease
}

\author{
James WT Kusena1', Robert J Thomas ${ }^{1}$, Mark J McCall ${ }^{1}$ \& Samantha L Wilson*,1 \\ ${ }^{1}$ Centre for Biological Engineering, Wolfson School of Mechanical, Electrical \& Manufacturing Engineering, Loughborough \\ University, Epinal Way, Loughborough, Leicestershire, LE11 3TU, UK \\ *Author for correspondence: Tel.: +44 150956 4866; S.Wilson2@lboro.ac.uk.
}

Current cell therapy product limitations include the need for in-depth product understanding to ensure product potency, safety and purity. New technologies require development and validation to address issues of production scale-up to meet clinical need; assays are required for process control, validation and release. Prior to clinical realization, an understanding of production processes is required to implement process changes that are essential for process control. Identification of key parameters forms the basis of process tolerances, allowing for validated, adaptive manufacturing processes. This enables greater process control and yield while withstanding regulatory scrutiny. This report summaries key milestones in specifically for ventral midbrain dopaminergic neuroprogenitor differentiation and key translational considerations and recommendations to enable successful, robust and reproducible current cell therapy product-manufacturing.

First draft submitted: 19 June 2019; Accepted for publication: 10 October 2019; Published online: 13 November 2019

Keywords: cell therapy $\bullet$ Parkinson's disease $\bullet$ process development $\bullet$ regenerative medicine $\bullet$ translation

The regenerative medicine and cell therapy industries are growing exponentially; both in terms of research and economic investment $[1,2]$. Their potential is not yet realized, partly due to the many process challenges developers encounter. Cell therapy products (CTPs) and regenerative medicine are inherently multidisciplinary and thus many aspects retard progress, from both process and manufacturing development standpoints. For the purpose of this review, the development of a CTP for the treatment of Parkinson's disease (PD) will be used as an exemplar study.

$\mathrm{PD}$ is hallmarked by the depletion of dopaminergic (DA) neurons in the substantia nigra pars compacta (SNc) of the midbrain, which produces the catecholamine neurotransmitter dopamine [3,4]. Dopamine is involved in neuromodulation, reward behaviors and motor utility of direct and indirect movement pathways [5]. Decreased DA density (specifically A8, A9 and A10 cells groups) and reduced dopamine results in diminished function of the nigrostriatal pathway and concomitant motor problems including hypokinesia, rigidity and tremors [4-9]. Additional motor-related symptoms experienced by PD patients include stooped posture, lack of facial expression, gait changes and dysphagia, while depression, dementia and sleep disturbances are typical nonmotor PD symptoms $[4,10]$.

\section{Therapies for the treatment of PD}

Current treatments for PD primarily concern dopamine, predominantly by introducing exogenous dopamine to increase levels within the brain, or preventing its degradation thus increasing bioavailability; or stimulating dopamine receptors via an agonistic effect. Akin to all drugs, PD drugs have several potential side effects, including: liver toxicity, sleep disturbances, hallucinations, delusions, dyskinesia and sometimes impulsive/compulsive behaviors [4]. In addition, prolonged use of medication therapy generally results in polypharmacy in order to ease the side effects of PD drugs such as anxiety and constipation [11]. As PD is progressive, pharmaceutical efficacy diminishes over time; resulting in increased dose strength and dosing frequency, which ultimately becomes inadequate as the symptoms become more pronounced. Furthermore, the therapeutic window in which patients are 'on' decreases, resulting in 
an increase of patient 'off' periods (a period in which the effect of the drug ceases prior to the next dose) and occurrences of dyskinesia caused by dosage increases [12].

Current trials have demonstrated that DA cell replacement can result in long-term survival ( $\geq 24$ years) of engrafted cells, which efficiently re-innervate the ventral mesencephalon into the dorsal striatum. This DA transplantation, engraftment and reinnervation mechanism of action (MOA) culminates in restored DA synthesis and release, providing long-term clinical improvement $\geq 14$ years, demonstrating proof-of-concept $[13,14]$. However, results are variable, despite many successes afforded for some of the patients that have undergone the transplantation procedures [15]. One major variant is the fetal material sourcing; highlighting requirements for optimized, standardized cell preparations. Fetal tissue-based therapies pose regulatory, reproducibility and ethical challenges [15-17]. Therefore, efforts are underway to produce ventral midbrain dopaminergic (vmDA) progenitors from sustainable and less ethically constrictive sources. This has resulted in protocol generation for human embryonic stem cells (hESC)-derived vmDA neuroprogenitors, with comparative efficacy and potency to fetal-derived equivalents [18].

It is important to note that transplantation trials have also been carried out using alternative cell sources, including induced pluripotent stem cells (iPSCs), parthenogenetic-derived DA neurons and neural stem cells [15,19,20]. However, the focus of this review will be hESC-derived cells due to their close relationship with current on-going research being carried out by the authors. Furthermore, the use of hESCs is pertinent since hESC-derived DA products are being developed commercially by companies, such as BlueRock Therapeutics, which was funded with vast amounts of money (US\$225 million) to progress CTP treatments [21]. BlueRock's pipeline includes a H9 hESC-derived DA progenitor product, which will be commencing first-in-human clinical trials this year $[19,22]$.

In comparison with iPSCs, hESCs face hurdles such as ethical and legal barriers restricting their use in some countries, for example France, Germany and the USA [23-25]. Furthermore, hESCs require human leukocyte antigen compatibility assessment, which is not necessary for patient derived iPSCs [26]. Nonetheless, hESCs are a cell type that have been studied for decades, unlike iPSCs which were introduced in 2006 [27]. There are still issues such as epigenetic and genetic aberrations, concomitant with the reprogramming process of iPSCs that needs to be addressed [26,28]. In addition, the DNA integrity of iPSCs and premature senescence of some iPSC-derived cells types has highlighted concerns regarding use of iPSC-derived products, in terms of their therapeutic safety and stability [29]. Moreover, in comparison with hESCs, iPSCs have been shown to produce reduced cell yields and increased variation in neuronal lineage differentiation processes [28,30], resulting in less consistency in the cell cultures. Thus, it is in the opinion of the authors that presently hESCs are a preferable cell source for vmDA transplantation, until iPSCs have been further studied in order to better demonstrate their genetic stability and safety. Further details regarding the comparisons of hESCs and iPSCs and their suitability as CTP cell sources can be found in reviews by Narsinh et al., Bilic and Belmonte and Bai et al.

An understanding of the developmental cues of the ventral mesencephalon, in particular, the cues that guide neurulation and mesencephalic development, has enabled the establishment of in vitro protocols that reliably produce transplantable vmDA neuroprogenitors cells. Refinement of these differentiation protocols has resulted in transplantable vmDA neuroprogenitors, derived from hESCs [16,31]. Hegarty et al. and Arenas et al. provides an indepth review and primer, respectively, offering comprehensive vmDA generation and development knowledge [32,33]. However, work is still required regarding product quality optimization and reproducible manufacturing processes. Therefore, rigorous characterization studies are required to ensure high safety and efficacy standards of the final product.

\section{In vitro differentiation of human embryonic stem cells into ventral midbrain dopaminaergic neurons}

Current cell replacement strategies are often hindered by ethical issues, reproducibility and cell source sustainability, availability and quality $[15,34,35]$. This has led to a plethora of work providing protocols to produce neural cell lineages, from embryonic and other pluripotent cell lines, for use in disease modeling and/or transplantation (Figure 1A) [36-38]. The resulting cells are similar to fetal cells, concerning morphology, surface antigen, protein expression, functionality, potency, growth and reinnervation [18,39,40].

The protocols examined in this review utilize hESCs. Other sources have been explored, including somatic cell reprogramming (using Lmx1a, Nurr1, Foxa2, En1 and Pitx3) [41,42]; however, these are out of scope of the present work. The main objective of a cell therapy applied to PD is to obtain DA progenitors. The identity of the progenitors is putatively marked by co-expression of the floor plate markers, FOXA2 and LMX1A, and the ability 


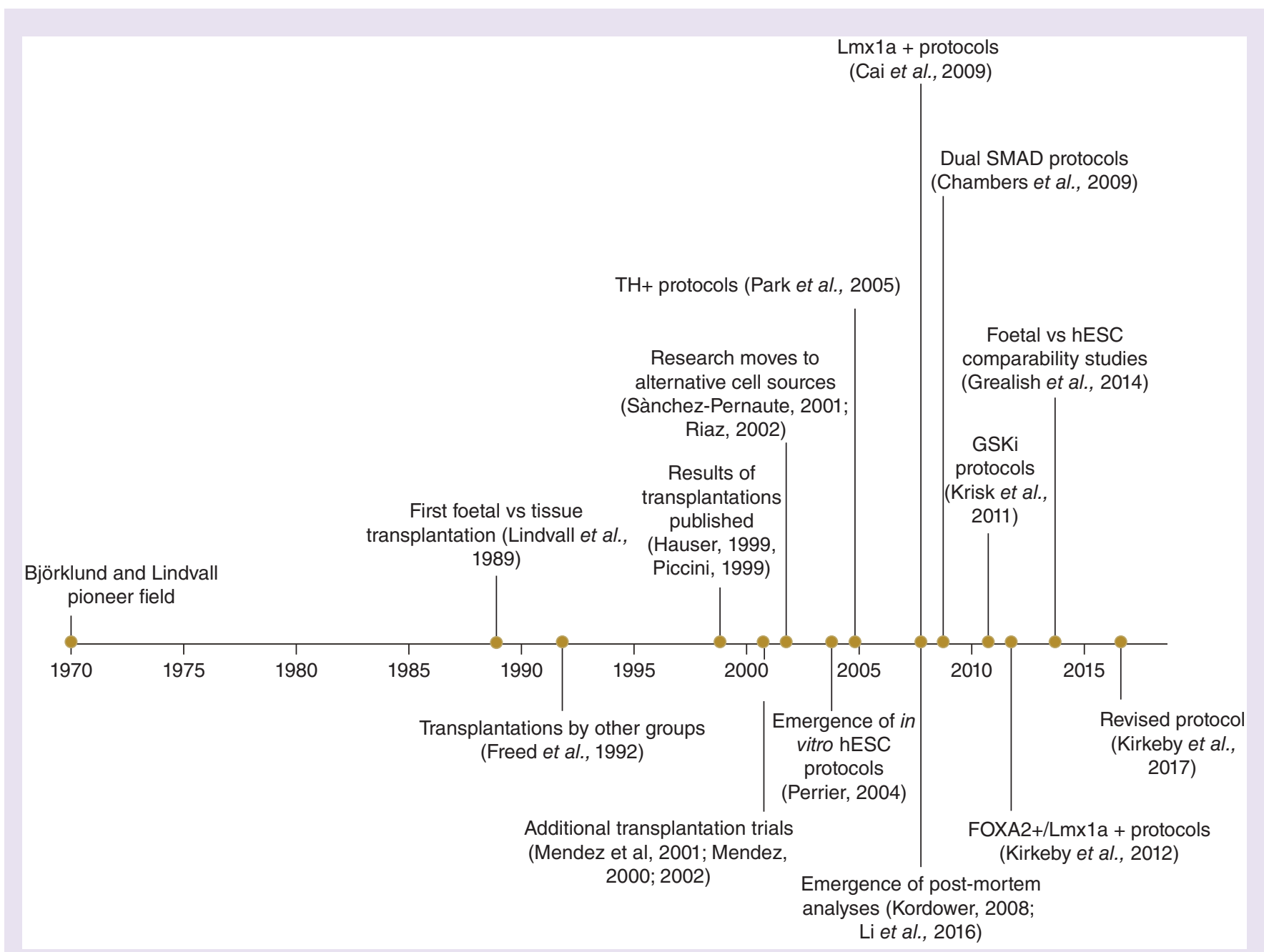

Figure 1. A timeline of ventral midbrain dopaminergic cell transplantation progression over five decades. Highlights include the first transplantations in 1987 and 1992; different protocols that have been developed to produce transplantable vmDA progenitors and the latest protocol from Lund University (Kirkeby et al.).

hESC: Human embryonic stem cells; vmDA: Ventral midbrain dopaminergic.

to produce dopamine, signified by expression of tyrosine hydroxylase (TH). Furthermore, the specific cell nuclei are critical, since several DA neuron groups exist, three of which (A8, A9 and A10) are found in the mesencephalon [43].

\section{The progression \& evolution of vmDA differentiation protocols}

Initial differentiation protocols focussed on the production of $\mathrm{TH}+$ cells since this was sufficient for dopamine synthesis $[44,45]$. Although seemingly successful, it was apparent that these cells were inadequately specific therapeutically, since other brain cells are $\mathrm{TH}+$ and produce dopamine. Typically, these protocols were from a FOXA2-/PAX6+ neural stem lineage grown from embryoid bodies (EBs) and feeder cells with activation of SHH and FGF-8 pathways to mimic embryogenesis $[41,42]$. However the resultant cells had reduced viability post-transplantation and formed nonspecific axonal outgrowth in rats [33], demonstrating that dopamine production $(\mathrm{TH}+)$ is not the sole requisite for therapeutic benefit, as the cells were not adequately specified into the vmDA A9 group [46-49].

Taking influence from embryogenesis, subsequent protocols were rigorous in recapitulating patterning factors and morphogens, facilitating more efficient vmDA neuroprogenitors. Chambers $e t$ al. introduced the important concept of the dual SMAD inhibition approach, which went on to form the basis of future neural differentiation protocols that followed [16,33,50-52]. This approach initially induces hESC neural induction, followed by patterning toward ventral mesencephalic fate [37]. The dual SMAD approach inhibits BMP and TGF- $\beta$, resulting in feeder- 


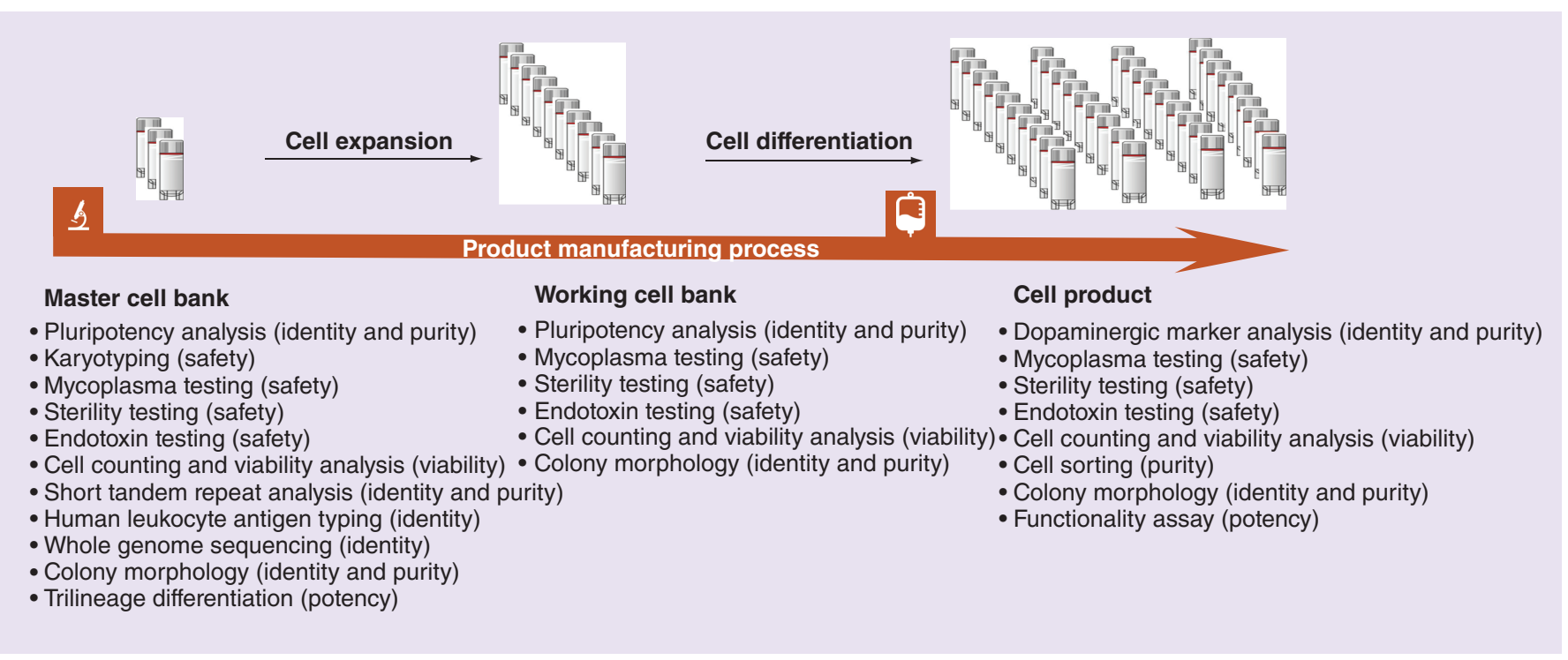

Figure 2. Illustrative product manufacturing timeline of a cell therapy product highlighting different assays that can be employed at different stages. The stages include a master cell bank that undergoes pluripotent cell expansion to create a working cell bank, which is then used to differentiate the cells into the cell product. The different assays address varying objectives such as assuring product purity, safety, identity, viability and potency.

free, efficient neural induction in the presence of RHO kinase inhibitor. The inhibition is dual, as blocking one pathway is insufficient for neural conversion. The addition of both noggin and SB 431542 blocks BMP and TGF- $\beta$ signaling, resulting in neuroectoderm differentiation by blocking SMAD transduction and decreasing pluripotency markers including Oct3/4 (POU5F1) [37].

Although the dual SMAD approach afforded neural induction, adjustments were necessary to ensure appropriate vmDA patterning trajectories. Kriks et al. demonstrated that the desired specificity into vmDA was incorrect as WNT $/ \beta$-catenin signaling was not initiated. The introduction of glycogen synthase kinase 3 inhibitor (GSK3i) into the in vitro differentiation process allowed for an iteration that activated the WNT/ $\beta$-catenin pathway, affording ventral-caudal patterning, resulting in midbrain cells with high co-localization of LMX1A and FOXA2 [53].

Since 2012, the dual SMAD and GSK3 $i$ approach has formed the basis of vmDA differentiation protocols [36,54]. Grealish $e t a l$. used this for preclinical studies and animal models, demonstrating vmDA-specified cells are capable of innervation without overgrowth or progeny $[18,55]$. The functional cells have long-term survival post-transplantation, produce dopamine and demonstrate improved motor behavior in rats [56,57]. Kirkeby et al. have since produced a series of hESC laminin-based protocol iterations using the dual SMAD approach and GSK3i. Dual SMAD and GSK3i protocols have produced in vitro yields $\geq 75 \%$ vmDA-identity cells, resulting in functional recovery of motor behavior in PD animals [33,37].

\section{Challenges \& considerations for vmDA nNeuron manufacturing translation}

Evidently mimicking embryogenesis is the most appropriate basis for contemporary hESC differentiation protocols. The challenges remaining concern addressing translational requirements to achieve robust manufacturing processes capable of meeting clinical need and scale. It is important that protocols can be reliably scaled-up using defined components and reagents to ensure good manufacturing practice compliance. This requires manufacturing process considerations to satisfy regulatory elements such as product quality and purity. This can be achieved by employing adequate process controls and validation assays, including karyotyping, phenotype analysis and endotoxin testing at appropriate time points throughout the manufacturing process (Figure 2) to characterize the product to ensure both efficacy and safety [58]. This is an onerous task with CTPs as they are a living entity and the cells themselves are the product and not just as part of the process, unlike to antibody production. The following sections highlight key considerations for process and protocol development that can translate efficiently and effectively into a manufactured CTP (Figure 3). 


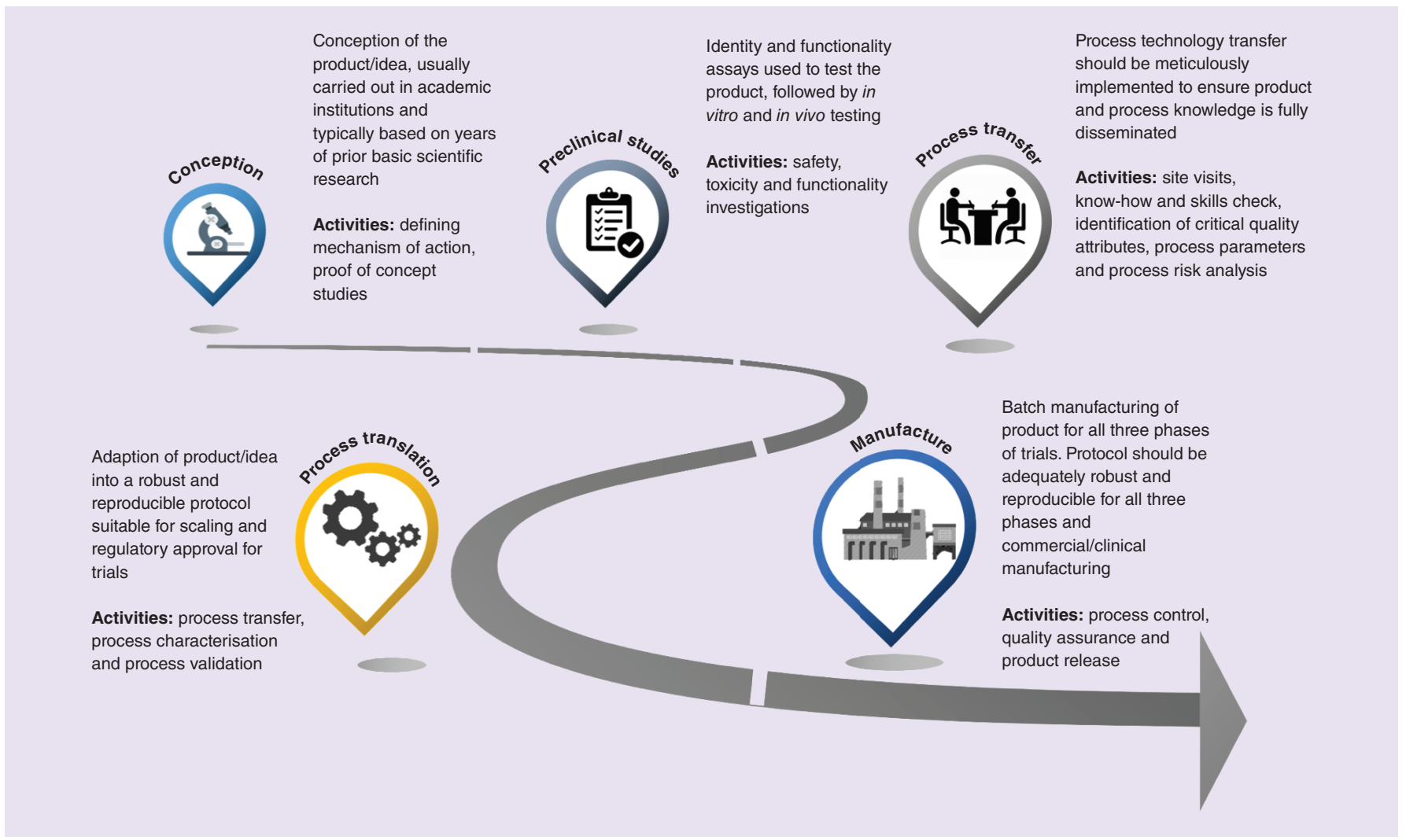

Figure 3. Process map of the key stages of cell therapy product development. Starting with conception of the product and ending with clinical or commercial product manufacture. The stages of conception and preclinical studies should be carried out with process translation and manufacture in mind; early consideration of the next steps can facilitate the generation of efficient, robust and well-informed protocols for CTP manufacture.

CTP: Cell therapy product.

\section{Purity}

Traditional pharmaceuticals assure high purity, however purity presents greater challenges for CTPs [59] due to the inherent living nature of cells, which interact with intrinsic and extrinsic factors. Unpredictable process variables pose problems where high purity yield governs functionality and efficacy. For instance, only vmDA would be required to ensure efficacy and innervation of the correct cell type into the appropriate regions of the dorsal striatum. Understandably, regulators expect proof of high purity, assuring that only the desired cells are procured for patients as undesired cell types may have a detrimental or unknown effect [60]. The challenge remains in achieving high purity with such complex products in other words, ensuring all cells are differentiated or manipulated to the desired state and, crucially, retain that state from bench to patient. This is important from a safety perspective and is scrutinized by regulators, since undesired cells may cause unwanted or unforeseen effects. This would be highly problematic and should be addressed through rigorous functionality testing and clear understanding of the MOA. Ideally, when a dossier is filed to the regulators purity, measures within the manufacturing process should be pre-established. In the case of vmDA manufacturing, appropriate cell identification and sorting assays, including magnetic-activated cell sorting and fluorescence-activated cell sorting should be considered and applied throughout the whole product development cycle, resulting in robustly validated purity release criteria for each batch. Furthermore, testing for the absence of bacteria, fungi and mycoplasma is essential; there are many techniques and organizations that can be employed to carry out such screening process [59,61,62]. In some cases, if accredited, in-house tests can be used to screen the product and this can be integrated into the quality management of the development and manufacturing process [63,64]. It is important to note that tests and interventions to remove specific pathogens must consider that cells are a living material and therefore cannot undergo rigorous sterilization and purification steps, such as irradiation, that occurs during the manufacturing process of traditional pharmaceuticals. 


\section{Identity}

Undesired cells (remnant pluripotent cells) remaining in the product when administered to the patient, may alter the MOA or result in spontaneous tumorigenesis. Cluster of differentiation markers are a prominent method of characterizing cells via surface molecules forming part of a product's target specification profile [62,65]. For instance, the co-expression of FOXA2 and OTX2 alludes to differentiation toward a vmDA cell lineage. However, like many aspects of CTPs, there are nuances presenting identification challenges, since similar cluster of differentiation marker combinations occur on very different cell types; or expression profiles are transient [66]. The European Medicines Agency (EMA) states "identity of cellular components should be based on phenotypic and/or genotypic markers" [67]. Consequently, test methods must be cell specific. When addressing phenotype, relevant analyses should include gene expression, antigen presentation and specific biochemical activity in an orthogonal manner [58,61]. For allogeneic CTPs, it is also imperative that identity profiles include histocompatibility markers, since cells will be scrutinized by the immune system. For vmDA cell transplantation, this is essential as the cells could be rejected, resulting in no innervation and inefficacy of the vmDA product.

\section{Tumorigenicity}

Tumorigenesis is a high risk potential for CTPs, particularly those using heavily manipulated cells or genetically edited cells. The transformation processes they undergo could result in chromosomal instability and genetic aberrations, consequently presenting an obstacle for regulatory authorization $[67,68]$. This relates back to the issue of purity, in the event that pluripotent cells are present in the end product, there is a risk that the cell would continue to proliferate and potentially become tumorigenic. For this reason, post-mitotic vmDAs are desired for transplantation, however the use of post- mitotic neuronal cells presents a dilemma in terms of decreased viability and integration of the cells. Thus, formulation of the cells and transplant cell numbers should adequately account for this, to ensure potency and efficacy of the product are retained. When using unestablished cell lines, karyology tests should be considered to investigate the tumorigenic potential of the cells as recommended by the EMA and US FDA [69], particularly when the cells themselves would be transplanted into the patients. The challenge is that CTPs may require stem cell use, extended cultures, banking and growth factors, all of which may result in tumorigenic profiles. The EMA states: "Use of cell lines known to be tumorigenic or to possess abnormal karyology should be evaluated in terms of risk-benefit for each product application" [69]. The FDA also employs a risk-based case-by-case approach to tumorigenicity analysis, which encourages for the most appropriate assays to be used that allow sufficient duration of time for any potential tumors to form.

\section{Potency}

According to the EMA, a cellular product should be able to demonstrate the intended biological effect that is applicable to its clinical therapeutic response [70]. Similarly, the FDA describes potency as the ability of a cellular product to provide a specific biological result that can be validated by appropriate laboratory analysis and clinical evidence [64]. Sometimes this is easily proven, however when potency tests must also show clinically meaningful changes it becomes difficult for some CTPs to demonstrate potency prior to human clinical trial data. For instance, in the case of PD, fluorodopa positron emission tomography is used to measure dopamine activity in patient; clinical changes in health utility are often measured by cognitive behavior assessments including the Unified PD Rating Scale and the Hoehn and Yahr scale. Due to the complex nature of CTPs, until this clinical data is available, developers may face unanswerable questions regarding product potency. However, in vitro and/or in vivo assays, or assays based on surrogate markers [61,62,64] including gene expression profiles and flow cytometry immunoassays can be employed to determine product potency. For example, dopamine release assays for vmDA neuroprogenitors can be used to ascertain potential dopamine levels; this information can be linked to the minimum dopamine levels required to provide improvements in motor symptoms. Such an assay would benefit from being nondestructive to the cells while having the ability to be employed in real time, as dopamine release would be a critical time point in the differentiation of vmDA. However, development of a potency assay for a CTP that is not at its functional end point presents many challenges. For instance with vmDA neuroprogenitors their axonal outgrowth cannot be measured using an assay prior to transplantation, without having to mature the cells. In addition, their potential to release dopamine cannot be measured at the progenitor stage; however, the use of markers such as TH could be used as a predictive indicator that the cells will produce dopamine within the dorsal striatum once engrafted. 


\section{Supply chain}

Supply chain reliability is often overlooked; however, reagents require batch validation with deviances being accounted for, or the demonstration of nondetrimental effects on the end product [61]. Therefore, it is important to consider reagents during process development to ensure manufacturing scalability. Furthermore, when assays come in contact with the product, it is essential that good manufacturing practice-compliant reagents are used as the end product will be in introduced into patients, and therefore it should be free of any harmful substances. Supply chain control and contingences are recommended to reduce variability, especially if tested within the design space [62]. This is pertinent for the differentiation of vmDA neuroprogenitors as the differentiation process requires a range of different supplemental and small molecule reagents. Suitably, these reagents should be simultaneously available or appropriate validated substitutes should be identified, to ensure that the differentiation process and product manufacturing is robust and amenable to supply chain changes. The latter activity may help overcome comparability issues further down the product timeline, especially if suppliers become obsolete. For supply chain management, product and assay development should be reliable and effective while considering associated costs, as hindrances to many CTPs are their cost-intensive development. This links to the need to consider reagent use and understanding the overall process unit costs [38].

\section{Quality by design; the importance of defining product characteristics}

The aforementioned challenges are addressed or well mitigated in well-established industries including antibody and pharmaceutical production, thus providing a learning platform for CTPs. An established manufacturing tool in pharmaceutical production is Juran's concept of Quality by Design (QbD). QbD facilitates product and process development that is data, risk and knowledge driven, with the intention of ensuring that quality is built into the product from the outset [71-73]. QbD key aspects include: defining quality target product profiles (QTPP) which are utilized to highlight the desired specifications, or critical quality attributes (CQAs), which ensure the desired product quality [72,74,75]. Having defined QTPP and CQAs precedes identification of critical material attributes and critical process parameters (CPPs) [74,76]. QbD concepts facilitate protocol and process development conceived with control strategies in place, ensuring that the QTPP is met in every production run; via identification and understanding the design space in which the CPPs result in the CQA and QTPP [74-76]. The design space helps developers attain process controls given the CPPs, and furthermore the use of process analytical technology (PAT) allows for iterative, data rich, informative development processes able to identify process capability [72,77].

GSK3 $i$ and SHH concentrations are integral CPPs for ventral mesencephalic patterning [33,78]. The lack of GSK3i and $\mathrm{SHH}$ results in dorsal fated neurons, while high concentrations result in hindbrain neurons; neither of these neuronal subtypes are TH+ [33]. Therefore, it is important to determine the appropriate concentrations of GSK3i and $\mathrm{SHH}$ to achieve the desired vmDA neuronal subtype [36,40,49,79]. Carrying out experimental investigations of the appropriate concentrations of these small molecules is essential to provide an understanding of the design space in which the desired CQAs can be obtained [74,76].

\section{Characterization}

Characterization is fundamental to CTP manufacturing; it is important to characterize and identify both input critical material attributes and output cells of a manufacturing process [62,80,81]. This facilitates input quality, ensuring materials are process appropriate; output characterization evaluates product manufacturing success [72,74,75]. CQAs are integral to product understanding and need defining prior to approval. CQAs are functionality-based characteristics, which can be physical or chemical attributes linked to potency, identity and/or purity [59,61,62]. These characteristics can be identified by cell morphology, phenotypic markers and secreted factors, which can be potentially linked to functionality and clinical response in other words, detection of secreted dopamine [62]. It is important that developers are selective and robust in their characterization, for instance distinct marker profiles should be used to confirm identity. This is crucial for CTPs such as vmDA progenitors for transplantation as subtle differences appear between DA neurons and neighboring neuronal cells. However, vmDA lineages restricted to the caudal ventral midbrain can be identified by FOXA2, LMX1A, CORIN, OTX2 and EN1 co-expression [18,33]. Therefore, using high specificity characterization criteria is a necessity for robust CTP assay development. Characterization during product development can provide validated and quantitative data applicable to potency assay development. Flow cytometry, genetic analysis and metabolite data are robust methods for analyzing identity, which can be used to track changes during expansion or differentiation. Correspondingly, potency assays based on MOA and cell characterization could be used to characterize products prerelease [18]. 


\section{Phenotypic markers}

Phenotypic markers can be used to ensure the CPPs and design space efficiently yield the desired CQAs. For instance, the small molecule concentrations and cell densities result in the desired cell markers and thus the CQAs in other words, a DA phenotype. Intracellular and extracellular markers can be transient, and thus are appropriate for monitoring process progression, including differentiation, using techniques such as flow cytometry or gene expression analysis. For a vmDA product, the input and output cell types have distinctive identity profiles. Positive expression of SSEA- 4 and Oct $3 / 4$ signifies undifferentiated cells, while decreased expression of these markers and transient presence of PAX6 demonstrates neural stem cell lineage progression [37]. The desired differentiated end product can be identified by FOXA2 and OTX2 co-expression [33]. Therefore, these markers can be assigned as CQAs since expression corresponds to specific stages, permitting development of in-process assays set against these CQAs. Furthermore, markers such as EN1 and TH have been linked to vmDA function in animal models.

\section{Technology}

Innovative adaption and transformation of technologies to meet new processes and product requirements is becoming commonplace. Instances include the creation of integrated systems resembling a modular process comprised of complementary technologies [65]. These aim to provide essential CTP monitoring capabilities, production control and production capacity to meet potential clinical demand. Scale up technologies are necessary for efficient manufacturing, however adequate control and monitoring technologies are equally essential for downstream manufacturing process optimization such as cell sorting, purification and filling [65]. Technologies such as fluorescence-activated cell sorting, automated cell counting and automated controlled rate freezing offer some solutions to ensure homogenous cell populations, ascertain cell viability and consistency of product manipulation on site, respectively [82-85].

Truly integrated inline systems, capable of monitoring, characterizing and sorting cells would allow for attainment of highly pure products, facilitating both process control and quality assurance. Furthermore, nondestructive PAT analyzing cell-secreted substances in real-time would allow for process mapping, as discrete secretome profiles are evident throughout differentiation. Failure to detect defined metabolites or secretome can act as a go/no-go decision tool for the differentiation and manufacturing processes, allowing for process interventions to evaluate whether the failure to detect metabolites or the secretome would impact the final product's quality and functionality. Furthermore, substance detection that is concomitant to product functionality in other words, secreted dopamine, could be a powerful potency assay. This can be achieved with PATs directly linked to the culture vessel that detect the desired substance in other words, metabolite analyzers, allowing for real-time process analysis and presenting a powerful tool for process development monitoring and CPP capability.

\section{Conclusion}

The growth of the cell therapy industries and the knowledge of vmDA differentiation suggests that CTPs for PD therapy is imminent. However, the translational space to bridge basic research into manufactured products is often neglected, which has retarded CTPs. A QbD mindset that fully characterizes a product throughout its life cycle and aids in the development of CQA informed in-process assays provides a powerful tool set for developers. This tool set provides product and process understanding which developers can use to address manufacturing challenges such as cell identification and assay development. A proactive stance to assay development is encouraged, considering how the product works and batch analysis at an early stage can mitigate risks during later stages of process and manufacturing development. A plethora of information demonstrating a clear understanding of the process and product is likely to favored by regulators, demonstrating due diligence throughout the development stages. An in-depth understanding of both process and product design spaces makes the product amenable to change, where necessary, negating further comparability studies and extensive paperwork. This permits informed, less hindered pathways to product regulatory authorization as 'early and sustained investment in a bonafide potency program is essential for maximizing a product's commercial success' [62].

\section{Future perspective}

Current CTP limitations include the need for in-depth product understanding to ensure product potency, safety and purity. New technologies require development and validation to address issues of production scale-up to meet clinical need; assays are required for process control, validation and release. Prior to clinical realization, an understanding of production processes is required to implement process changes which is essential for process control. Identification of key parameters forms the basis of process tolerances, allowing for validated, adaptive 
manufacturing processes. This enables greater process control and yield while withstanding regulatory scrutiny. The application of these key translational considerations and recommendations will enable successful, robust and reproducible CTP-manufacturing.

Executive summary

Therapies for the treatment of Parkinson's disease

- The development of robust protocols for the production of ventral midbrain dopaminergic neuron (vmDA) progenitor cells for the treatment of Parkinson's disease has been used as an exemplar case in this review.

- Transplantation of vmDA neuroprogenitors has been shown to successfully reverse symptoms of Parkinson's disease. However, current research protocols are presently not optimized into reproducible, robust manufacturing processes.

In vitro differentiation of human embryonic stem cells into vmDA neurons

- Successful protocol derivation is dependent upon a prior knowledge and understanding of developmental in vivo cellular cues and processes.

Challenges \& considerations for vmDA neuron manufacturing translation

- Despite significant research and economic growth in the field of regenerative medicine and cell therapy industries the full potential has not yet been; realized this has in part been retarded by both process and manufacturing stances.

- In order to be fully translational and clinically relevant, cell therapy products (CTPs) must be reliably manufactured and scaled up using defined good manufacturing practice-compliant processes, components and reagents.

Quality by design, the importance of defining product characteristics

- Manufacturing processes must satisfy strict regulatory requirements including purity, validity and safety. Employing quality by design from the outset ensures that process development is data, risk and knowledge driven, thus ensuring quality CTPs.

- Protocol and process development must be conceived with control strategies in place to ensure that quality target profiles are met every time. Characterization and defined critical quality attributes are fundamental to this, in terms of both cellular input and output.

\section{Conclusion}

- An in-depth understanding of process and product design enables cellular products to be amenable to change, which negates the need for further comparability studies and extensive paperwork.

- A proactive stance to assay development is vital, this includes a consideration of how the product works and the inclusion of early-stage batch analysis to mitigate risks during later stages of process and manufacturing development.

Financial \& competing interests disclosure

The authors acknowledge financial support from the Engineering and Physical Sciences Research Council (grant no. EP/L015072/1). The authors have no other relevant affiliations or financial involvement with any organization or entity with a financial interest in or financial conflict with the subject matter or materials discussed in the manuscript apart from those disclosed.

No writing assistance was utilized in the production of this manuscript.

\section{Open access}

This work is licensed under the Creative Commons Attribution 4.0 License. To view a copy of this license, visit http://creativecomm ons.org/licenses/by/4.0/

\section{References}

Papers of special note have been highlighted as: $\bullet$ of interest; $\bullet \bullet$ of considerable interest

1. Mason C, Brindley DA, Culme-Seymour EJ, Davie NL. Cell therapy industry: billion dollar global business with unlimited potential. Regen. Med. 6(3), 265-272 (2011).

2. Mason C, McCall MJ, Culme-Seymour EJ et al. The global cell therapy industry continues to rise during the second and third quarters of 2012. Cell Stem Cell 11(6), 735-739 (2012).

3. Kalia LV, Lang AE, Lees AJ, Hardy J, Revesz T. Parkinson's disease. Lancet 386(9996), 896-912 (2015).

4. Jankovic J. Parkinson's disease: clinical features and diagnosis. J. Neurol. Neurosurg. Psychiatry 79(4), 368-376 (2008).

5. Hagell P, Crabb L, Pogarell O et al. Health-related quality of life following bilateral intrastriatal transplantation in Parkinson's disease. Mov. Disord. 15(2), 224-229 (2000). 
6. Cenci MA, Lundblad M. Post- versus presynaptic plasticity in L-DOPA-induced dyskinesia. J. Neurochem. 99(2), 381-392 (2006).

7. Soh SE, Mcginley J, Morris ME. Measuring quality of life in Parkinson's disease: selection of-an-appropriate health-related quality of life instrument. Physiotherapy 97(1), 83-89 (2010).

8. Reuther M, Spottke EA, Klotsche J et al. Assessing health-related quality of life in patients with Parkinson's disease in a prospective longitudinal study. Park. Relat Disord. 13(2), 108-114 (2007).

9. Hoehn MM, Yahr MD. Parkinsonism: onset, progression, and mortality. Neurology 17(5), 427-442 (1967).

10. Chin JH, Vora N. The global burden of neurologic diseases. Neurology 83(4), 349-351 (2014).

11. Sveinbjornsdottir S. The clinical symptoms of Parkinson's disease. J. Neurochem. 139, 318-324 (2016).

12. Pahwa R, Lyons KE. Levodopa-related wearing-off in Parkinson's disease: identification and management. Curr. Med. Res. Opin. 25(4), 841-849 (2009).

13. Kordower JH, Chu Y, Hauser RA, Freeman TB, Olanow CW. Lewy body-like pathology in long-term embryonic nigral transplants in Parkinson's disease. Nat. Med. 14(5), 504-506 (2008).

14. Li W, Englund $\mathrm{E}$, Widner $\mathrm{H}$ et al. Extensive graft-derived dopaminergic innervation is maintained 24 years after transplantation in the degenerating parkinsonian brain. Proc. Natl Acad. Sci. USA 113(23), 6544-6549 (2016).

15. Parmar M, Torper O, Drouin-Ouellet J. Cell-based therapy for Parkinson's disease: a journey through decades toward the light side of the Force. Eur. J. Neurosci. 49(4), 463-471 (2019).

16. Nolbrant S, Heuer A, Parmar M, Kirkeby A. Generation of high-purity human ventral midbrain dopaminergic progenitors for in vitro maturation and intracerebral transplantation. Nat. Protoc. 12(9), 1962-1979 (2017).

- Detailed protocol of how to derive ventral midbrain dopaminergic (DA) progenitors, which has been used by a range of institutions including the authors and it is the protocol of interest for the TransEuro clinical trial programe.

17. Studer L. Strategies for bringing stem cell-derived dopamine neurons to the clinic - The NYSTEM trial. Prog. Brain Res. 230, 191-212 (2017).

18. Grealish S, Diguet E, Kirkeby A et al. Human ESC-derived dopamine neurons show similar preclinical efficacy and potency to fetal neurons when grafted in a rat model of Parkinson's disease. Cell Stem Cell 15(5), 653-665 (2014).

- Highlights that human embryonic stem cells are similar to fetal neurons, thus they are a credible source of functional ventral midbrain DA progenitors for transplantation into humans.

19. Irion S. Cell therapies for Parkinson's disease. Clin. Transl. Sci. 12(2), 95-97 (2019).

- Highlights the cell therapies clinical trials currently on going for the treatment of Parkinson's disease.

20. Barker RA. TRANSEURO consortium. Designing stem-cell-based dopamine cell replacement trials for Parkinson's disease. Nat. Med. 25(7), 1045-1053 (2019).

21. Bayer AG. Bayer and versant ventures join forces to launch stem cell therapy company bluerock therapeutics with USD 225 million series a financing - bayer news (2016). https://media.bayer.com/baynews/baynews.nsf/id/Bayer-Versant-Ventures-Join-Forces-Launch -Stem-Cell-Therapy-Company-BlueRock-Therapeutics-USD

22. BlueRock Therapeutics. Pipeline-Realizing the potential of cellular and gene therapy engineered cell therapy (2019). https://bluerocktx.com/pipeline/

23. Young FE. A time for restraint. Science 287(5457), 1424 (2000).

24. Lenoir N. Europe confronts the embryonic stem cell research challenge. Science 287(5457), 1425-1427 (2000).

25. European Commission, Secretariat of the European Group on Ethics in Science and New Technologies. European Group on Ethics in Science and New Technologies; human tissue banks; human embryo research. Hum. Reprod. Genet. Ethics. 5(1), 1-19 (1999).

26. Bai Q, Desprat R, Klein B, Lemaitre J-M, De Vos J. Embryonic stem cells or induced pluripotent stem cells? A DNA integrity perspective. Curr. Gene Ther. 13(2), 93-98 (2013).

27. Takahashi K, Yamanaka S. Induction of pluripotent stem cells from mouse embryonic and adult fibroblast cultures by defined factors. Cell 126(4), 663-676 (2006).

28. Wu JC. Comparison of human induced pluripotent and embryonic stem cells: fraternal or identical twins? Mol. Ther. 19(4), 635-638 (2011).

29. Bilic J, Belmonte JCI. Concise review: induced pluripotent stem cells versus embryonic stem cells: close enough or yet too far apart? Stem Cells 30(1), 33-41 (2012).

30. Hu B-Y, Weick JP, Yu J et al. Neural differentiation of human induced pluripotent stem cells follows developmental principles but with variable potency. Proc. Natl Acad. Sci. USA 107(9), 4335-4340 (2010).

31. Kirkeby A, Parmar M, Barker RA. Strategies for bringing stem cell-derived dopamine neurons to the clinic: a European approach (STEM-PD). Prog. Brain Res. 230, 165-190 (2017).

32. Hegarty SV, Sullivan AM, O'Keeffe GW. Midbrain dopaminergic neurons: a review of the molecular circuitry that regulates their development. Dev. Biol. 379(2), 123-138 (2013). 
-. Provides in depth understanding of the development of DA neurons.

33. Arenas E, Denham M, Villaescusa JC. How to make a midbrain dopaminergic neuron. Development 142(11), 1918-1936 (2015).

- Reviews of the protocols that are used to obtain DA progenitors and the different iterations that have been made to the protocols.

34. Stoker TB, Barker RA. Cell therapies for Parkinson's disease: how far have we come? Regen. Med. 11(8), 777-786 (2016).

- Provides a good overview of the progress that has been made regarding the use and development of cell therapies for the treatment of Parkinson's disease.

35. Stoker TB, Blair NF, Barker RA. Neural grafting for Parkinson’s disease: challenges and prospects. Neural Regen. Res. 12(3), 389-392 (2017).

36. Kirkeby A, Nelander J, Parmar M. Generating regionalized neuronal cells from pluripotency, a step-by-step protocol. Front. Cell. Neurosci. 6(64), 1-4 (2013).

37. Chambers SM, Fasano CA, Papapetrou EP, Tomishima M, Sadelain M, Studer L. Highly efficient neural conversion of human ES and iPS cells by dual inhibition of SMAD signaling. Nat. Biotechnol. 27(3), 275-280 (2009).

-• Discusses the use of the dual SMAD inhibition approach to neural differentiation which has become the basis of many neural differentiation protocols since.

38. Kirkeby Agnete PM. Building authentic midbrain dopaminergic neurons from stell cells - lessons from development. Transl. Neurosci. 3(4), 314-319 (2012).

39. Kirkeby A, Nolbrant S, Tiklova K et al. Predictive markers guide differentiation to improve graft outcome in clinical translation of hESC-based therapy for Parkinson's disease. Cell Stem Cell 20(1), 135-140 (2017).

40. Kirkeby A, Grealish S, Wolf DA et al. Generation of regionally specified neural progenitors and functional neurons from human embryonic stem cells under defined conditions. Cell Rep. 1(6), 703-714 (2012).

41. Kikuchi T, Morizane A, Doi D et al. Human iPS cell-derived dopaminergic neurons function in a primate Parkinson's disease model. Nature 548(7669), 592-596 (2017).

42. Schilling H, Feng J, Chu X, Xu Z, Chen S, Jiang H. Induced dopaminergic neurons: a new promise for Parkinson's disease. Redox Biol. 11, 606-612 (2017).

43. German DC, Manaye KF. Midbrain dopaminergic neurons (nuclei A8, A9, and A10): three-dimensional reconstruction in the rat. J. Comp. Neurol. 331(3), 297-309 (1993).

44. Sundberg M, Bogetofte H, Lawson T et al. Improved cell therapy protocols for Parkinson's disease based on differentiation efficiency and safety of hESC-, hiPSC-, and non-human primate iPSC-derived dopaminergic neurons. Stem Cells 31(8), 1548-1562 (2013).

45. Hartfield EM, Yamasaki-Mann M, Fernandes HJR et al. Physiological characterisation of human iPS-derived dopaminergic neurons. PLoS ONE 9(2), e87388 (2014).

46. Kawasaki H, Mizuseki K, Nishikawa S et al. Induction of midbrain dopaminenergic neurotechnique neurons from ES cells by stromal cell-derived inducing activity. Neuron 28(1), 31-40 (2000).

47. Kim J-H, Auerbach JM, Rodriguez-Gómez JA et al. Dopamine neurons derived from embryonic stem cells function in an animal model of Parkinson's disease. Nature 418(6893), 50-56 (2002).

48. Roy NS, Cleren C, Singh SK, Yang L, Beal MF, Goldman SA. Functional engraftment of human ES cell-derived dopaminergic neurons enriched by coculture with telomerase-immortalized midbrain astrocytes. Nat. Med. 12(11), 1259-1268 (2006).

49. Bruses J, Rubio ME, Topf $\mathrm{N}$ et al. Derivation of midbrain dopamine neurons from human embryonic stem cells. Proc. Natl Acad. Sci. USA 101(34), 12543-12548 (2004).

50. Tomishima M. Neural induction - Dual SMAD inhibition. StemBook Girard L (Ed). Harvard Stem Cell Institute, MA, USA (2012).

51. Wattanapanitch M, Klincumhom N, Potirat P et al. Dual small-molecule targeting of SMAD signaling stimulates human induced pluripotent stem cells toward neural lineages. PLoS ONE 9(9), e106952 (2014).

52. Grealish S, Diguet E, Kirkeby A et al. Human ESC-derived dopamine neurons show similar preclinical efficacy and potency to fetal neurons when grafted in a rat model of Parkinson's disease. Cell Stem Cell 15(5), 653-665 (2014).

53. Kriks S, Shim J-W, Piao J et al. Floor plate-derived dopamine neurons from hESCs efficiently engraft in animal models of PD HHS public access. Nature 480(7378), 547-551 (2012).

54. Denham M, Bye C, Leung J, Conley BJ, Thompson LH, Dottori M. Glycogen synthase kinase $3 \beta$ and activin/nodal inhibition in human embryonic stem cells induces a pre-neuroepithelial state that is required for specification to a floor plate cell lineage. Stem Cells 30(11), 2400-2411 (2012).

55. Kirkeby A, Nolbrant $\mathrm{S}$, Tiklova $\mathrm{K}$ et al. Predictive markers guide differentiation to improve graft outcome in clinical translation of hESC-based therapy for Parkinson's disease. Cell Stem Cell 20(1), 135-148 (2017).

56. Wakeman DR, Hiller BM, Marmion DJ et al. Cryopreservation maintains functionality of human iPSC dopamine neurons and rescues Parkinsonian phenotypes in vivo. Stem Cell Rep. 9(1), 149-161 (2017).

57. Heuer A, Kirkeby A, Pfisterer U, Jönsson ME, Parmar M. hESC-derived neural progenitors prevent xenograft rejection through neonatal desensitisation. Exp. Neurol. 282, 78-85 (2016). 
58. European Medicines Agency. Guideline on human cell-based medicinal products (2008). https://www.ema.europa.eu/en/documents/scientific-guideline/guideline-human-cell-based-medicinal-products_en.pdf

59. Lipsitz YY, Timmins NE, Zandstra PW. Quality cell therapy manufacturing by design. Nat. Biotechnol. 34(4), 393-400 (2016).

-• Discusses the use of quality by design as a tool for dealing with the challenges of cell therapy manufacturing, in particular how quality by design can be used to ascertain final product quality.

60. Bubela T, McCabe C, Archibald P et al. Bringing regenerative medicines to the clinic: the future for regulation and reimbursement. Regen. Med. 10(7), 897-911 (2015).

- Discusses the regulatory considerations for regenerative medicines, highlighting the need to demonstrate quality, safety and efficacy.

61. Carmen J, Burger SR, McCaman M, Rowley Ja. Developing assays to address identity, potency, purity and safety: cell characterization in cell therapy process development. Regen. Med. 7(1), 85-100 (2012).

62. Bravery CA, Carmen J, Fong $\mathrm{T}$ et al. Potency assay development for cellular therapy products: an ISCT* review of the requirements and experiences in the industry. Cytotherapy 15(1), 9-19 (2013).

63. U.S. Department of Health and Human Services, Food and Drug Administration. Guidance for Industry Preclinical Assessment of Investigational Cellular and Gene Therapy Products (2013). https://www.fda.gov/regulatory-information/search-fda-guidance-docum ents/preclinical-assessment-investigational-cellular-and-gene-therapy-products

64. U.S. Department of Health and Human Services, Food and Drug Administration. Guidance for Industry: Potency Tests for Cellular and Gene Therapy Products (2011). https:

//www.fda.gov/regulatory-information/search-fda-guidance-documents/potency-tests-cellular-and-gene-therapy-products

65. Brindley DA, French AL, Baptista R et al. Cell therapy bioprocessing technologies and indicators of technological convergence. Bioprocess Int. 12(3), 14-21 (2014).

66. Holmes N. CD45: all is not yet crystal clear. Immunology 117(2), 145-155 (2006).

67. European Medicines Agency. Guideline on human cell-based medicinal products (2007). https://www.ema.europa.eu/en/documents/scientific-guideline/draft-guideline-human-cell-based-medicinal-products_en.pdf

68. Salmikangas P, Menezes-Ferreira M, Reischl I et al. Manufacturing, characterization and control of cell-based medicinal products: challenging paradigms toward commercial use. Regen. Med. 10(1), 65-78 (2015).

69. European Medicines Agency. ICH Topic Q5D quality of biotechnological products: derivation and characterisation of cell substrates used for production of biotechnological/biological products (1997). https://www.ema.europa.eu/en/documents/scientific-guideline/ich -q-5-d-derivation-characterisation-cell-substrates-used-production-biotechnological/biological-products-step-5_en.pdf

70. European Medicines Agency. Adoption by chmp for release for consultation (2007). http://www.emea.europa.eu

71. Juran JM. Juran on quality by design: the new steps for planning quality into goods and services. The Free Press, NY, USA (1992).

72. Yu LX, Amidon G, Khan MA et al. Understanding pharmaceutical quality by design. AAPS J. 16(4), 771-783 (2014).

73. Rathore AS, Winkle H. Quality by design for biopharmaceuticals. Nat. Biotechnol. 27(1), 26-34 (2009).

74. Politis SN, Colombo P, Colombo G, Rekkas DM. Design of experiments (DoE) in pharmaceutical development. Drug Dev. Ind. Pharm. 43(6), 889-901 (2017).

75. Huang J, Goolcharran C, Ghosh K. A quality by design approach to investigate tablet dissolution shift upon accelerated stability by multivariate methods. Eur. J. Pharm. Biopharm. 78(1), 141-150 (2011).

76. Huang J, Kaul G, Cai C et al. Quality by design case study: an integrated multivariate approach to drug product and process development. Int. J. Pharm. 382(1), 23-32 (2009).

77. Thomas RJ, Hourd PC, Williams DJ. Application of process quality engineering techniques to improve the understanding of the in vitro processing of stem cells for therapeutic use. J. Biotechnol. 136(3), 148-155 (2008).

78. Xi J, Liu Y, Liu H, Chen H, Emborg ME, Zhang SC. Specification of midbrain dopamine neurons from primate pluripotent stem cells. Stem Cells 30(8), 1655-1663 (2012).

79. Cai J, Donaldson A, Yang M, German MS, Enikolopov G, Iacovitti L. The role of Lmx1a in the differentiation of human embryonic stem cells into midbrain dopamine neurons in culture and after transplantation into a Parkinson's disease model. Stem Cells 27(1), 220-229 (2009).

80. French A, Bravery C, Smith J et al. Enabling consistency in pluripotent stem cell-derived products for research and development and clinical applications through material standards. Stem Cells Transl. Med. 4(3), 217-223 (2015).

81. Pimpaneau V, Gianelli F, Trouvin JH, Poiseau AD. The challenges of potency assay development for cell-based medicinal products in Europe. Regul. Rapp. 12(5), 5-10 (2015).

82. Doi D, Samata B, Katsukawa M et al. Isolation of human induced pluripotent stem cell-derived dopaminergic progenitors by cell sorting for successful transplantation. Stem Cell Rep. 2(3), 337-350 (2014).

83. Massie I, Selden C, Hodgson H, Fuller B, Gibbons S, Morris GJ. GMP cryopreservation of large volumes of cells for regenerative medicine: active control of the freezing process. Tissue Eng. Part C Methods 20(9), 693-702 (2014). 
84. Ware CB, Nelson AM, Blau CA. Controlled-rate freezing of human ES cells. BioTechniques 38(6), 879-883 (2005).

85. Shu Z, Kang X, Chen $\mathrm{H}$ et al. Development of a reliable low-cost controlled cooling rate instrument for the cryopreservation of hematopoietic stem cells. Cytotherapy 12(2), 161-169 (2010). 